\title{
A Recursive Demodulator for Real-Time Measurement of Multiple Sinusoids
}

DOI:

10.1109/JSEN.2018.2844398

\section{Document Version}

Accepted author manuscript

Link to publication record in Manchester Research Explorer

\section{Citation for published version (APA):}

Sun, S., Xu, L., Cao, Z., Sun, J., \& Yang, W. (2018). A Recursive Demodulator for Real-Time Measurement of Multiple Sinusoids. IEEE Sensors Journal, 18(15), 6281-6289. https://doi.org/10.1109/JSEN.2018.2844398

\section{Published in:}

IEEE Sensors Journal

\section{Citing this paper}

Please note that where the full-text provided on Manchester Research Explorer is the Author Accepted Manuscript or Proof version this may differ from the final Published version. If citing, it is advised that you check and use the publisher's definitive version.

\section{General rights}

Copyright and moral rights for the publications made accessible in the Research Explorer are retained by the authors and/or other copyright owners and it is a condition of accessing publications that users recognise and abide by the legal requirements associated with these rights.

\section{Takedown policy}

If you believe that this document breaches copyright please refer to the University of Manchester's Takedown Procedures [http://man.ac.uk/04Y6Bo] or contact uml.scholarlycommunications@manchester.ac.uk providing relevant details, so we can investigate your claim.

\section{OPEN ACCESS}




\title{
A Recursive Demodulator for Real-time Measurement of Multiple Sinusoids
}

\author{
Shijie Sun, Member, IEEE, Lijun Xu*, Senior Member, IEEE, Zhang Cao, Member, IEEE, Jiangtao Sun, Member, \\ IEEE, and Wuqiang Yang, Fellow, IEEE
}

\begin{abstract}
A fast demodulator for real-time measurement of the amplitudes and phases of multiple sinusoids is highly desired in many applications. When the sampling frequency is fixed, the number of samples needed for demodulation determines the time consumption and hence the demodulation speed. In this paper, a quadrature demodulation method is firstly presented, which requires that the samples cover exactly integer periods of the multi-frequency signal. Secondly, a new recursive demodulation method is proposed, which can not only overcome the limitation of the quadrature method, but also provide greater flexibility between accuracy and speed, i.e. either to obtain a higher speed at an expense of a lower accuracy, or vice versa. The proposed method can work with a sample series of any length as long as more than twice the number of sinusoids and generate demodulation results for all sinusoids simultaneously. Thirdly, a resource-saving and fast implementation of the recursive demodulator was proposed and constructed on a DSP/FPGA, which enables in-situ and on-line application of the recursive demodulator. Experiments were carried out to investigate the performance of the proposed demodulator, including relative error, standard deviation and their variations with the number of samples involved in the demodulation, and compare with other conventional methods.
\end{abstract}

Index Terms-Recursive demodulator, Quadrature method, Demodulation speed, DSP/FPGA

\section{INTRODUCTION}

$\mathrm{I}^{\mathrm{n}}$ the active measurement of various object parameters, an external excitation signal is applied on the target object through a sensor. By measuring the response signal that carries the information of the object's characteristic parameters, the parameters to be measured can be derived. Sinusoid is a kind of commonly used excitation signal. To quickly obtain the target parameters of the object under excitation of a series of given frequencies, multiple sinusoids of the given frequencies can be combined as an excitation signal so that the unknowns are measured simultaneously. Because the response sensor signal is also composed of multiple sinusoids with the same frequencies and the frequencies are pre-known, only the amplitudes and phases of

This work was supported by the National Natural Science Foundation of China (No. 61620106004 and 61522102) and National Postdoctoral Program for Innovative Talents (No. BX201700021).

S. Sun, L. Xu, Z. Cao, and J. Sun are with the Key Laboratory of Precision Opto-Mechatronic Technology of Ministry of Education, the School of Instrument Science and Opto-Electronic Engineering, Beihang University, Beijing, 100191, China (e-mails: lijunxu@buaa.edu.cn and zh_cao@buaa.edu.cn).

W. Yang is with the School of Electrical and Electronic Engineering, the University of Manchester, UK. the sinusoids need to be obtained. The active measurement is required in many industrial and biomedical applications, such as industrial process tomography [1]-[3], bio-impedance measurement [4]-[6], eddy current testing [7], [8] and built-in self-test [9], etc. Because the characteristic parameters of the target object are often rapidly changing, real-time and fast measurement of the amplitudes and phases of multiple sinusoids in the response sensor signal is highly desired in sensor data processing.

The demodulation process can be implemented using an analog method [10], which consists of multiplexers and filters. However, the demodulation speed is restricted by the highorder low-pass filters. In recent years, digital demodulators have been widely used, including the synchronous sampling method [11], the DFT, FFT [12]-[14] and quadrature methods [15]-[18], etc. The synchronous sampling method is low-cost but the demodulation speed is also restricted by the low sampling rate. The DFT method converts a finite-length sequence of equally-spaced samples into an equivalent-length sequence of complex values, which carries the information of the amplitude and phase of each frequency component. To reduce spectrum leakage and obtain accurate demodulation results, the samples in a complete or integer periods of the signal are required. The main drawback of the DFT method is its heavy computation burden. The FFT method is used to reduce the computing complexity of the DFT and provides exactly the same results as the DFT method. Both the DFT and FFT methods require the samples in a complete or integer periods of a multi-frequency signal to accurately demodulate the multiple sinusoids, resulting in a limited demodulation speed. The main principle of the quadrature method is quite similar to the DFT method and it also requires that the samples cover integer periods of the multi-frequency signal.

The drawbacks of the DFT, FFT and quadrature methods are (1) we have to wait for one or several periods of the excitation signal for data sampling to obtain an estimate of the required parameters, and (2) we cannot obtain an accurate estimate using the sampled data of non-integer periods, e.g. smaller than one complete period.

These drawbacks of the traditional methods have limited the demodulation speed. To deal with the problem that the quadrature method requires one complete or integer periods of a sinusoid for demodulation, a recursive least squares method [19] and an information-filtering method [1], [20] were ever proposed for the demodulation of a singlefrequency signal by the research group of the authors. Each of the two methods can output a preliminary demodulation 
result using the first two samples although the accuracy is very low. The accuracy can then be recursively improved by involving more and more samples. Because the two methods do not require the samples to cover integer signal periods, tradeoffs between speed and accuracy can be achieved. When a high speed is required, samples within part of one complete signal period may be used, although at an expense of a lower accuracy.

If we could use the recursive method to demodulate multiple sinusoids, it will greatly improve the demodulation speed. However, extending the recursive method from single sinusoid demodulation to multiple sinusoids demodulation is still a challenging task. Compared with single sinusoid demodulation, the number of parameters to be measured in the multiple sinusoids is much larger and the number of samples used for the demodulation has a greater influence on the demodulation accuracy. In this case, a more sophisticated mathematical model is required and the computation complexity will increase consequently, leading to much more resource consumption, which is critical to real-time application when using a digital signal processor (DSP) or a field-programmable gate array (FPGA).

Aiming to solve the above problems, we developed a new recursive model for multiple sinusoids demodulation to simultaneously obtain the amplitudes and phases of multiple sinusoids for active measurement applications. By dividing the implementation process into pre-computing and onlinecomputing stages, a resource-saving and fast implementation based on a DSP or FPGA is proposed, which enables in-situ and on-line application of the recursive demodulator.

This paper is organized as follows. In Section II, the quadrature demodulation method is first formulized, and then two recursive demodulation methods are proposed. In Section III, a fast recursive demodulator was constructed based on a DSP or FPGA in a resource-saving manner and the resources consumption of the traditional methods and the proposed methods are analyzed and compared. Section IV is devoted to evaluate the performance of the proposed demodulator and compare with that of the traditional methods experimentally. Section V concludes the research outcomes.

\section{Methodology}

\section{A. Demodulation of Multiple Sinusoids}

Let's assume a signal with $M$ sinusoidal components as

$$
y(t)=\sum_{m=1}^{M} A_{m} \cos \left(\omega_{m} t+\varphi_{m}\right)+\varepsilon(t),
$$

where $A_{m}, \omega_{m}$ and $\varphi_{m}$ are the amplitude, angular frequency and phase of the $m$-th sinusoidal component of the signal $y$, and $\varepsilon$ is the measurement noise associated with $y$.

When the signal is sampled by an ADC with a sampling frequency of $f_{s}$, its discretized form is

$$
y(n)=\sum_{m=1}^{M} A_{m} \cos \left(\omega_{m} \frac{n}{f_{s}}+\varphi_{m}\right)+\varepsilon(n), \quad n=1,2, \ldots,
$$

where $n$ is the serial number of the sample, $\omega_{m}$ and $f_{m}$ are the angular frequency and frequency of the $m$-th sinusoidal component, respectively, and $\omega_{m}=2 \pi f_{m}$.

Equation (2) can be rewritten in a matrix form:

$$
y(n)=\boldsymbol{u}_{n} \cdot \boldsymbol{x}+\varepsilon(n),
$$

where $\boldsymbol{u}_{n}$ is the measurement vector that can be obtained from the known information of the sinusoidal components, $\boldsymbol{x}$ is the state vector related to the amplitudes and phases of all sinusoidal components of the signal. $\boldsymbol{u}_{n}$ and $\boldsymbol{x}$ are expressed as

$$
\boldsymbol{u}_{n}=\left[\begin{array}{c}
\cos \left(\omega_{1} \frac{n}{f_{s}}\right) \\
\cos \left(\omega_{2} \frac{n}{f_{s}}\right) \\
\vdots \\
\cos \left(\omega_{M} \frac{n}{f_{s}}\right) \\
\sin \left(\omega_{M} \frac{n}{f_{s}}\right) \\
\vdots \\
\sin \left(\omega_{2} \frac{n}{f_{s}}\right) \\
\sin \left(\omega_{1} \frac{n}{f_{s}}\right)
\end{array}\right]^{T}, \boldsymbol{x}=\left[\begin{array}{c}
A_{1} \cos \left(\varphi_{1}\right) \\
A_{2} \cos \left(\varphi_{2}\right) \\
\vdots \\
A_{M} \cos \left(\varphi_{M}\right) \\
-A_{M} \sin \left(\varphi_{M}\right) \\
\vdots \\
-A_{2} \sin \left(\varphi_{2}\right) \\
-A_{1} \sin \left(\varphi_{1}\right)
\end{array}\right],
$$

where the operator []$^{\mathrm{T}}$ denotes the transpose.

When $N$ samples are obtained, the following set of $N$ equations is obtained.

$$
\boldsymbol{Y}_{N}=\boldsymbol{U}_{N} \cdot \boldsymbol{x}+\boldsymbol{\varepsilon},
$$

where $\boldsymbol{Y}$ is the vector of samples, $\boldsymbol{U}$ is the measurement matrix, $\boldsymbol{\varepsilon}$ is the error vector and

$$
\left\{\begin{array}{l}
\boldsymbol{Y}_{N}=\left[\begin{array}{llll}
y(1) & y(2) & \ldots & y(N)
\end{array}\right]^{T} \\
\boldsymbol{U}_{N}=\left[\begin{array}{llll}
\boldsymbol{u}_{1} & \boldsymbol{u}_{2} & \ldots & \boldsymbol{u}_{N}
\end{array}\right]^{T} \\
\boldsymbol{\varepsilon}_{N}=\left[\begin{array}{llll}
\varepsilon(1) & \varepsilon(2) & \ldots & \varepsilon(N)
\end{array}\right]^{T}
\end{array},\right.
$$

For a specific $N$, the elements of $\boldsymbol{U}$ are fixed, which are only determined by $N$, the frequencies of sinusoidal components of the signal and the sampling rate of the ADC. If $\boldsymbol{U}$ is invertible, $\boldsymbol{x}$ can be obtained from (5) by:

$$
\boldsymbol{x}=\boldsymbol{U}_{N}^{-1} \cdot\left(\boldsymbol{Y}_{N}-\boldsymbol{\varepsilon}\right),
$$

where $\boldsymbol{U}_{N}^{-1}$ is the inverse of $\boldsymbol{U}_{N}$.

With the state vector $\boldsymbol{x}$, the amplitudes and phases of the sinusoidal components of the signal can be calculated by

$$
\left\{\begin{array}{c}
A=\left[\begin{array}{c}
A_{1} \\
A_{2} \\
\vdots \\
A_{M}
\end{array}\right]=\left[\begin{array}{c}
\sqrt{\boldsymbol{x}(1)^{2}+\boldsymbol{x}(2 M)^{2}} \\
\sqrt{\boldsymbol{x}(2)^{2}+\boldsymbol{x}(2 M-1)^{2}} \\
\vdots \\
\sqrt{\boldsymbol{x}(M)^{2}+\boldsymbol{x}(M+1)^{2}}
\end{array}\right], \\
\varphi=\left[\begin{array}{c}
\varphi_{1} \\
\varphi_{2} \\
\vdots \\
\varphi_{M}
\end{array}\right]=\left[\begin{array}{c}
\arccos \left[\boldsymbol{x}(1) / A_{1}\right] \\
\arccos \left[\boldsymbol{x}(2) / A_{2}\right] \\
\vdots \\
\arccos \left[\boldsymbol{x}(M) / A_{M}\right]
\end{array}\right]
\end{array}\right.
$$

where $\boldsymbol{x}(m)$ is the $m$-th element of $\boldsymbol{x}$. Then equation (7) needs to be solved to obtain the state vector $\boldsymbol{x}$ for signal demodulation. 


\section{B. Least Squares Solution and Quadrature Demodulation Method}

In (7), the state vector $\boldsymbol{x}$ cannot be calculated directly because the inverse of $\boldsymbol{U}$ does not exist when more than $2 M$ samples are involved. In this case, the least squares method can be used to obtain an estimate of $\boldsymbol{x}$ :

$$
\boldsymbol{x}=\left(\boldsymbol{U}_{N}{ }^{T} \boldsymbol{U}_{N}\right)^{-1} \boldsymbol{U}_{N}{ }^{T}\left(\boldsymbol{Y}_{N}-\boldsymbol{\varepsilon}_{N}\right) .
$$

However, when the number of sinusoidal components of the signal is large, it is difficult to calculate $\boldsymbol{x}$ using (9).

Regardless of the error term, when $N$ samples are used, substituting (6) into (9) results in:

$$
\begin{aligned}
& \boldsymbol{x}=\left(\begin{array}{cccc}
\sum_{n=1}^{N} \cos ^{2}\left(\omega_{1} \frac{n}{f_{s}}\right) & \sum_{n=1}^{N} \cos \left(\omega_{1} \frac{n}{f_{s}}\right) \cos \left(\omega_{2} \frac{n}{f_{s}}\right) & \cdots & \sum_{n=1}^{N} \cos \left(\omega_{1} \frac{n}{f_{s}}\right) \sin \left(\omega_{1} \frac{n}{f_{s}}\right) \\
\sum_{n=1}^{N} \cos \left(\omega_{2} \frac{n}{f_{s}}\right) \cos \left(\omega_{1} \frac{n}{f_{s}}\right) & \sum_{n=1}^{N} \cos ^{2}\left(\omega_{2} \frac{n}{f_{s}}\right) & \cdots & \sum_{n=1}^{N} \cos \left(\omega_{2} \frac{n}{f_{s}}\right) \sin \left(\omega_{1} \frac{n}{f_{s}}\right) \\
\vdots & \vdots & \vdots & \vdots \\
\sum_{n=1}^{N} \sin \left(\omega_{1} \frac{n}{f_{s}}\right) \cos \left(\omega_{1} \frac{n}{f_{s}}\right) & \sum_{n=1}^{N} \sin \left(\omega_{1} \frac{n}{f_{s}}\right) \cos \left(\omega_{2} \frac{n}{f_{s}}\right) & \cdots & \sum_{n=1}^{N} \sin ^{2}\left(\omega_{1} \frac{n}{f_{s}}\right)
\end{array}\right)^{-1} \\
& {\left[\begin{array}{lllll}
\sum_{n=1}^{N} \cos \left(\omega_{1} \frac{n}{f_{s}}\right) y(n) & \cdots & \sum_{n=1}^{N} \cos \left(\omega_{M} \frac{n}{f_{s}}\right) y(n) \sum_{n=1}^{N} \sin \left(\omega_{M} \frac{n}{f_{s}}\right) y(n) & \cdots & \sum_{n=1}^{N} \sin \left(\omega_{1} \frac{n}{f_{s}}\right) y(n)
\end{array}\right]^{t}}
\end{aligned}
$$

In (10), the individual sinusoidal components of angular frequencies $\omega_{1}, \omega_{2}, \ldots \omega_{M}$, are required and the sinusoidal components of combined frequencies $\omega_{\alpha}+\omega_{\beta}$ and $\omega_{\alpha}-\omega_{\beta}(\alpha, \beta=1,2 \cdots M)$, are unwanted. According to the orthogonality of trigonometric functions, when the sampled data cover exactly integer periods of each sinusoidal component of the multi-frequency signal, the unwanted sinusoidal components can be removed.

$$
\left\{\begin{array}{l}
\sum_{n=1}^{N} \cos \left(\omega_{\alpha} \frac{n}{f_{s}}\right) \cos \left(\omega_{\beta} \frac{n}{f_{s}}\right)= \begin{cases}\frac{N}{2} & (\alpha=\beta \text { and } \alpha, \beta=1,2 \cdots M \\
0 & (\alpha \neq \beta \text { and } \alpha, \beta=1,2 \cdots M\end{cases} \\
\sum_{n=1}^{N} \sin \left(\omega_{\alpha} \frac{n}{f_{s}}\right) \sin \left(\omega_{\beta} \frac{n}{f_{s}}\right)= \begin{cases}\frac{N}{2} & (\alpha=\beta \text { and } \alpha, \beta=1,2 \cdots M) \\
0 & (\alpha \neq \beta \text { and } \alpha, \beta=1,2 \cdots M)\end{cases} \\
\sum_{n=1}^{N} \cos \left(\omega_{\alpha} \frac{n}{f_{s}}\right) \sin \left(\omega_{\beta} \frac{n}{f_{s}}\right)=0 \quad(\alpha, \beta=1,2 \cdots M)
\end{array}\right.
$$

Then the state vector $\boldsymbol{x}$ becomes

$$
\boldsymbol{x}=\frac{2}{N}\left[\sum_{n=1}^{N} \cos \left(\omega_{1} \frac{n}{f_{s}}\right) \quad \cdots \quad \sum_{n=1}^{N} \cos \left(\omega_{M} \frac{n}{f_{s}}\right) \quad \sum_{n=1}^{N} \sin \left(\omega_{M} \frac{n}{f_{s}}\right) \quad \cdots \quad \sum_{n=1}^{N} \sin \left(\omega_{1} \frac{n}{f_{s}}\right)\right] y(n)
$$

The in-phase and quadrature-phase items can be obtained by

$$
\left\{\begin{aligned}
I_{m} & =\frac{2}{N} \sum_{n=1}^{N} \cos \left(\omega_{m} \frac{n}{f_{s}}\right) \cdot y(n) \\
& =\frac{2}{N} \sum_{n=1}^{N} \frac{A_{m}}{2}\left(\cos \left(2 \omega_{m} \frac{n}{f_{s}}+\varphi_{m}\right)+\cos \left(\varphi_{m}\right)\right)=A_{m} \cos \left(\varphi_{m}\right) \\
Q_{m} & =\frac{2}{N} \sum_{n=1}^{N} \sin \left(\omega_{m} \frac{n}{f_{s}}\right) \cdot y(n) \\
& =\frac{2}{N} \sum_{n=1}^{N} \frac{A_{m}}{2}\left(\sin \left(2 \omega_{m} \frac{n}{f_{s}}+\varphi_{m}\right)+\sin \left(-\varphi_{m}\right)\right)=-A_{m} \sin \left(\varphi_{m}\right)
\end{aligned}\right.
$$

The accumulation process is equivalent to a low-pass filter to reduce the measurement noises. Then the amplitudes and phases of the signal's sinusoidal components can be calculated by

$$
\left\{\begin{array}{l}
A_{m}=\sqrt{I_{m}^{2}+Q_{m}^{2}} \\
\varphi_{m}=\arccos \left[\frac{I_{m}}{A_{m}}\right] \quad(m=1,2, \cdots M) .
\end{array}\right.
$$

This quadrature demodulation method requires the samples covering integer periods of each sinusoidal component of the multi-frequency signal. Therefore, the demodulation speed is limited by the periods or frequencies of the signal's sinusoidal components.

\section{Fourier Transform Methods}

The DFT method can convert a finite-length sequence of samples, i.e. $y(1), y(2), \ldots y(N)$, into an equivalent-length sequence of complex values, i.e. $X(1), X(2), \ldots X(N)$, which carry the information of the amplitude and phase of each frequency component, i.e. $2 \pi f_{s} n / N(1 \leqslant n \leqslant N)$. The DFT results can be expressed as

$$
X(k)=D F T[y(n)]=\sum_{n=1}^{N} y(n) \mathrm{e}^{-j \frac{2 \pi}{N} n k}(1 \leq k \leq N, 1 \leq n \leq N) .
$$

To reduce spectrum leakage and obtain more accurate demodulation results, the samples in a complete or integer periods of the signal are required in the DFT method. Then (15) can be expressed as

$$
\left[\begin{array}{c}
X(1) \\
X(2) \\
\vdots \\
X(N)
\end{array}\right]=\left[\begin{array}{cccc}
e^{-j \frac{2 \pi}{N} \times 1 \times 1} & e^{-j \frac{2 \pi}{N} \times 2 \times 1} & \cdots & e^{-j \frac{2 \pi}{N} \times N \times 1} \\
e^{-j \frac{2 \pi}{N} \times 1 \times 2} & e^{-j \frac{2 \pi}{N} \times 2 \times 2} & \cdots & e^{-j \frac{2 \pi}{N} \times N \times 2} \\
\vdots & \vdots & \cdots & \vdots \\
e^{-j \frac{2 \pi}{N} \times 1 \times N} & e^{-j \frac{2 \pi}{N} \times 2 \times N} & \cdots & e^{-j \frac{2 \pi}{N} \times N \times N}
\end{array}\right]\left[\begin{array}{c}
y(1) \\
y(2) \\
\vdots \\
y(N)
\end{array}\right] .
$$

If $f_{s} k / N=f_{m}, X(k)$ for the frequency component, $f_{m}$, can be calculated by

$$
\begin{aligned}
& X_{m}(k)=\left[\begin{array}{c}
e^{-j \frac{2 \pi}{N} \times x n} \\
e^{-\frac{2 \pi}{N} \times 2 \times n} \\
\vdots \\
e^{-j \frac{2 \pi}{N} \times N \times n}
\end{array}\right]^{T}\left[\begin{array}{cc}
\cos \left(\omega_{m} \frac{1}{f_{s}}\right) & \sin \left(\omega_{m} \frac{1}{f_{s}}\right) \\
\cos \left(\omega_{m} \frac{2}{f_{s}}\right) & \sin \left(\omega_{m} \frac{2}{f_{s}}\right) \\
\vdots & \vdots \\
\cos \left(\omega_{m} \frac{N}{f_{s}}\right) & \sin \left(\omega_{m} \frac{N}{f_{s}}\right)
\end{array}\right]\left[\begin{array}{c}
A_{m} \cos \left(\varphi_{m}\right) \\
-A_{m} \sin \left(\varphi_{m}\right)
\end{array}\right] \\
& =\left[\begin{array}{l}
\sum_{n=1}^{N} \cos \left(\omega_{m} \frac{n}{f_{s}}\right) \cos \left(\omega_{m} \frac{n}{f_{s}}\right)-j \sum_{n=1}^{N} \sin \left(\omega_{m} \frac{n}{f_{s}}\right) \cos \left(\omega_{m} \frac{n}{f_{s}}\right) \\
\sum_{n=1}^{N} \cos \left(\omega_{m} \frac{n}{f_{s}}\right) \sin \left(\omega_{m} \frac{n}{f_{s}}\right)-j \sum_{n=1}^{N} \sin \left(\omega_{m} \frac{n}{f_{s}}\right) \sin \left(\omega_{m} \frac{n}{f_{s}}\right)
\end{array}\right]^{T}\left[\begin{array}{c}
A_{m} \cos \left(\varphi_{m}\right) \\
-A_{m} \sin \left(\varphi_{m}\right)
\end{array}\right] \\
& =\frac{N}{2}\left(A_{m} \cos \left(\varphi_{m}\right)+j A_{m} \sin \left(\varphi_{m}\right)\right)=\frac{N}{2}\left(I_{m}-j Q_{m}\right)
\end{aligned}
$$

Then the amplitudes and phases of the multiple sinusoids can be calculated by (14). It can be seen that the demodulation process using the DFT method for a specific frequency component is actually the same as the quadrature demodulation method. When the frequencies of multiple sinusoids are pre-known, the quadrature demodulation method is more cost-efficient than the DFT method because only the specific frequency components are required to be measured. 
The FFT method is used to reduce the computing complexity of the DFT, which can provide the same result as the DFT method. For a radix-2 FFT method, only when the sampling frequency is $L 2^{N}$ (where $L$ and $N$ are two integers, $L$ is the number of signal periods and $2^{N}$ is the number of samples in one complete signal period) times of the signal frequency, the phases and amplitudes of the sinusoids can be accurately estimated. For other sampling frequencies, spectrum leakage will cause an demodulation error.

\section{Recursive Demodulation Method}

Theoretically, the amplitudes and phases of the $M$ sinusoidal components can be obtained using the first $2 M$ samples when the frequencies are known. However, the demodulation accuracy is low due to the sample noises. To improve the accuracy, more samples are needed to reduce the effect of sample noises. This paper focuses on how to obtain the amplitudes and phases of the sinusoidal components using a sampled data series of any length as long as more than $2 M$. Two methods are introduced to demodulate a multi-frequency signal using samples in non-integer signal periods, especially less than one complete signal period.

Firstly, the conventional recursive least squares (RLS) method is used to solve (9). In the RLS method, $\boldsymbol{x}$ can be recursively estimated by

$$
\begin{gathered}
G_{n}=J_{n-1} \boldsymbol{u}_{n}^{\mathrm{T}}\left[R_{n}+\boldsymbol{u}_{n} J_{n-1} \boldsymbol{u}_{n}^{T}\right]^{-1}, \\
J_{n}=J_{n-1}-G_{n} \boldsymbol{u}_{n} J_{n-1}, \\
\boldsymbol{x}_{n}=\boldsymbol{x}_{n-1}+G_{n}\left(y(n)-\boldsymbol{u}_{n} \boldsymbol{x}_{n-1}\right),
\end{gathered}
$$

where $G_{n}$ is the gain in the $n$-th update, $R$ is the correlation matrix of measurement noise, and $J$ is the correlation matrix of the error of $\boldsymbol{x}$. The RLS method provides a recursive way to solve the least squares problem. However, when the RLS method is implemented in a DSP or FPGA for real-time demodulation, it is very resource-consuming.

To solve this problem, the information-filter (IF) method [21] is used to solve (9) and a new recursive form is proposed in the following. The inverse state-error correlation matrix is defined firstly as

$$
K_{n}=\left[\begin{array}{llll}
\boldsymbol{u}_{1} & \boldsymbol{u}_{2} & \cdots & \boldsymbol{u}_{n}
\end{array}\right]\left[\begin{array}{llll}
\boldsymbol{u}_{1} & \boldsymbol{u}_{2} & \cdots & \boldsymbol{u}_{n}
\end{array}\right]^{T} .
$$

Then the $n$-th update of $\boldsymbol{K}$ can be obtained from

$$
\begin{aligned}
K_{n} & =\left[\begin{array}{llll}
\boldsymbol{u}_{1} & \boldsymbol{u}_{2} & \cdots & \boldsymbol{u}_{n}
\end{array}\right]\left[\begin{array}{llll}
\boldsymbol{u}_{1} & \boldsymbol{u}_{2} & \cdots & \boldsymbol{u}_{n}
\end{array}\right]^{T} \\
& =\left[\begin{array}{llll}
\boldsymbol{u}_{1} & \boldsymbol{u}_{2} & \cdots & \boldsymbol{u}_{n-1}
\end{array}\right]\left[\begin{array}{llll}
\boldsymbol{u}_{1} & \boldsymbol{u}_{2} & \cdots & \boldsymbol{u}_{n-1}
\end{array}\right]^{T}+\boldsymbol{u}_{n}^{T} \boldsymbol{u}_{n}
\end{aligned} .
$$

Therefore, $\boldsymbol{K}$ is updated by

$$
K_{n}=K_{n-1}+\boldsymbol{u}_{n}^{T} \boldsymbol{u}_{n} .
$$

The $n$-th estimate of $\boldsymbol{x}$ is

$$
\begin{aligned}
\boldsymbol{x}_{n} & =\left(K_{n}\right)^{-1}\left[\begin{array}{llll}
\boldsymbol{u}_{1} & \boldsymbol{u}_{2} & \cdots & \boldsymbol{u}_{n}
\end{array}\right]\left[\begin{array}{llll}
y(1) & y(2) & \cdots & y(n)
\end{array}\right]^{T} \\
& =\left(K_{n}\right)^{-1}\left[\begin{array}{c}
\boldsymbol{u}_{1} \\
\boldsymbol{u}_{2} \\
\vdots \\
\boldsymbol{u}_{n-1}
\end{array}\right]^{T}\left[\begin{array}{c}
y(1) \\
y(2) \\
\vdots \\
y(n-1)
\end{array}\right]+\left(K_{n}\right)^{-1} \boldsymbol{u}_{n}^{T} y(n) \\
& =\left(K_{n}\right)^{-1}\left(K_{n-1} \boldsymbol{x}_{n-1}\right)+\left(K_{n}\right)^{-1} \boldsymbol{u}_{n}^{T} y(n)
\end{aligned}
$$

Let's assume

$$
P_{n}=K_{n} x_{n} .
$$

Then $P_{n}$ is updated by the new samples as

$$
P_{n}=P_{n-1}+\boldsymbol{u}_{n}^{T} y(n) .
$$

Substituting (4) into (26) results in

$$
\left[\begin{array}{c}
P_{n}(1) \\
P_{n}(2) \\
\vdots \\
P_{n}(2 M)
\end{array}\right]=\left[\begin{array}{c}
P_{n-1}(1) \\
P_{n-1}(2) \\
\vdots \\
P_{n-1}(2 M)
\end{array}\right]+\left[\begin{array}{c}
\cos \left(\omega_{1} \frac{n}{f_{s}}\right) \\
\cos \left(\omega_{2} \frac{n}{f_{s}}\right) \\
\vdots \\
\sin \left(\omega_{1} \frac{n}{f_{s}}\right)
\end{array}\right] \cdot y(n),
$$

where $P_{n}(m)$ is the $m$-th element of $P_{n} . \boldsymbol{x}$ can be calculated by

$$
\boldsymbol{x}_{n}=\left(K_{n}\right)^{-1}\left(K_{n} \boldsymbol{x}_{n}\right)=\left(K_{n}\right)^{-1} P_{n} .
$$

Finally, the amplitude and phase of each sinusoidal component of the signal can be obtained by (8).

To start the recursion process, the parameters $K$ and $P$ should be initialized, which would affect the starting value of $\boldsymbol{x}$. The initial values of $K$ and $P$ can be set as fixed values, but if they are far away from their true values, the converging process will be slow. To improve the demodulation accuracy and convergence speed, the initial values of $K$ and $P$ are initialized using the first $2 M$ samples as

$$
\left\{\begin{array}{l}
K_{2 M}=\left(\boldsymbol{U}_{2 M}\right)^{T} \boldsymbol{U}_{2 M} \\
P_{2 M}=\left(\boldsymbol{U}_{2 M}\right)^{T} Y_{2 M}
\end{array} .\right.
$$

Then the first estimate of $\boldsymbol{x}$ can be obtained by

$$
\boldsymbol{x}_{2 M}=\left(K_{2 M}\right)^{-1} P_{2 M} .
$$

The amplitudes and phases of the sinusoidal components can be further calculated by (8).

If the measurement noises are Gaussian, stable and with zero mean, the RLS method and IF method are mathematically equivalent. From Section II. $B$, we can further know that when the samples covering exactly integer periods of the signal are used for demodulation, the RLS and IF methods are equivalent to the quadrature demodulation method.

By comparing the two recursive methods, equations (20) and (27) are the main recursive equations which are calculated by involving new samples. The multiplication and addition operations of the IF method, as in (20), are much less than those of the RLS method, as in (27), which makes the IF method more resource-saving in DSP/FPGA implementation.

\section{REAL-TIME IMPLEMENTATION}

\section{A. Implementation in DSP/FPGA}

To achieve real-time signal demodulation, the demodulation method is generally required to be implemented in a DSP or FPGA, which is a popular processor in many measurement systems, such as the electrical tomography system. Here, a resource-saving and fast implementation of the recursive demodulation method is 
proposed. Taking the IF method as an example, the implementation procedure has three steps:

1) Off-line step: Equation (23) can be calculated in advance because the parameters in (23) are pre-known and no new samples are involved. The updating results, $K^{-1}$, can be stored in a PC for Step 3;

2) Updating step: Equation (27) needs to be updated using $\boldsymbol{u}_{n}$ and the newly coming samples and should be calculated online. The elements of $\boldsymbol{u}_{n}$, including $\sin \left(\omega_{m} n / f_{s}\right)$ and $\cos \left(\omega_{m} n / f_{s}\right) \quad(n=1 \cdots N, m=1 \cdots M)$, can be calculated using a look-up table;

3) Output step: Equation (28) is used to output $\boldsymbol{x}$ using $K^{-1}$ and $P$ from the first two steps. This step can be calculated in the PC.

By analyzing the implementation procedure, it can be seen that only the updating step needs to be calculated online in the DSP or FPGA. For each update, $2 M$ operations of multiplication are required, i.e., $\cos \left(\omega_{m} n / f_{s}\right) y(n)$ and $\sin \left(\omega_{m} n / f_{s}\right) y(n)(m=1 \cdots M)$. If $N$ samples are used in the updating step, the results of the last update need to be transmitted to the PC for the output step, i.e.

$$
\boldsymbol{x}_{N}=\left(K_{N}\right)^{-1} P_{N} \text {. }
$$

The implementing process of the IF demodulator using the DSP or FPGA is shown in Fig 1. The detailed steps to implement the IF method are as follows:

\begin{tabular}{|c|c|c|}
\hline Start & Step 1) & $\begin{array}{l}\text { Determine the number of samples for } \\
\text { demodulation, } N \text {, according to the } \\
\text { required demodulation accuracy; }\end{array}$ \\
\hline Offline & Step 2) & $\begin{array}{l}\text { Compute } \boldsymbol{K}^{-1} \text { and store the results in the } \\
\text { PC using the given excitation } \\
\text { frequencies } f_{1}, f_{2}, \ldots, f_{M} \text { and the } \\
\text { sampling rate } f_{s} \text {, and then calculate the } \\
\text { values of } \cos \left(\omega_{m} n / f_{s}\right) \text { and } \\
\sin \left(\omega_{m} n / f_{s}\right)(m=1 \cdots M) \text { offline and } \\
\text { store the values in look-up tables in the } \\
\text { DSP/FPGA; }\end{array}$ \\
\hline $\begin{array}{l}\text { DSP/ } \\
\text { FPGA }\end{array}$ & Step 4) & $\begin{array}{l}\text { Initialize the parameter } \boldsymbol{P} \text { by (29); } \\
\text { Update (27) using the newly coming } \\
\text { samples and the look-up tables; } \\
\text { Stop the updating step and transmit the } \\
\text { values of } P_{N} \text { to the PC when the } \\
\text { number of samples reaches } N \text {; }\end{array}$ \\
\hline PC & Step 6) & $\begin{array}{l}\text { Calculate } \boldsymbol{x}_{N} \text { by }(31) \text {; } \\
\text { Calculate the amplitudes and phases of } \\
\text { all sinusoidal components of the signal } \\
\text { by }(8) \text {. }\end{array}$ \\
\hline
\end{tabular}

It should be noted that it is difficult to perform floatingpoint calculations in a FPGA, which is good at fixed-point calculations. Therefore, when the IF method is implemented in a FPGA, the values of $\cos \left(\omega_{m} n / f_{s}\right)$ and $\sin \left(\omega_{m} n / f_{s}\right)$ $(m=1 \cdots M)$ should be converted to fixed-point values and then store the values in the look-up tables. As shown in
Section II. $D$, the implementation process of the RLS method is very similar to the IF method.

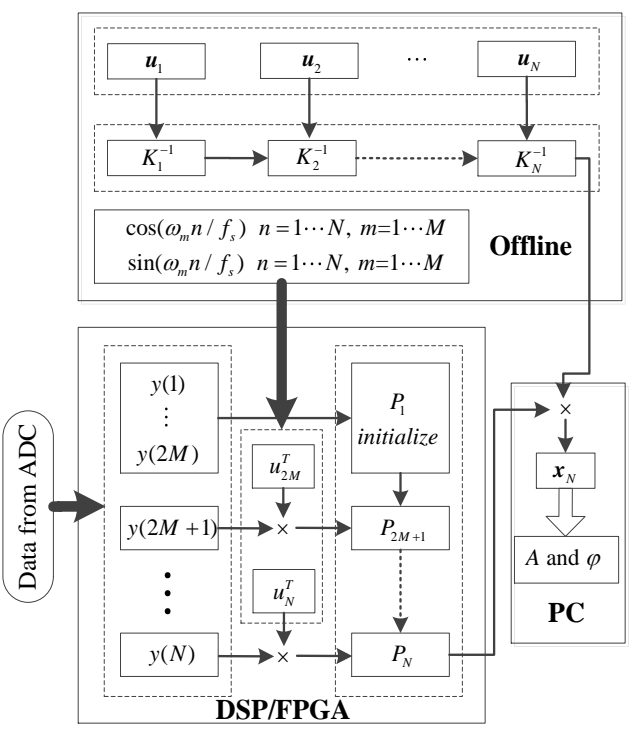

Fig. 1 IF method implemented in DSP/FPGA

\section{B. Comparision of Resources Consumption}

Using the above method, the recursive demodulation methods are implemented in a DSP (TMS320C6713B, TI) and a FPGA (EP3C25Q, Altera), respectively. When $N$ samples are used for demodulation, the numbers of multiplications and additions required to be operated in DSP/FPGA using different methods are listed in Table I.

TABLE I

COMPARISON OF RESOURCES CONSUMPTION AMONG DIFFERENT METHODS

\begin{tabular}{cccc}
\hline \hline Methods & & $\begin{array}{c}\text { Number of } \\
\text { multiplications }\end{array}$ & $\begin{array}{c}\text { Number of } \\
\text { additions }\end{array}$ \\
\hline Non-recursive & QD & $2 M N$ & $2 M(N-1)$ \\
demodulation & DFT & $N^{2}$ & $N(N-1)$ \\
& FFT & $N / 2 \cdot \log _{2} N$ & $N \log _{2} N$ \\
\hline Recursive & RLS & $\left(8 M^{2}+6 M+1\right) N$ & $8 M^{2}(N-1)$ \\
demodulation & IF & $2 M N$ & $2 M(N-1)$ \\
\hline \hline
\end{tabular}

The above methods can be divided into two types:

1) Recursive methods, including the RLS and IF methods

Compared with the RLS method, the IF method requires less multiplication operations, and hence saves hardware resources. Here, it should be noted that when the number of samples used for demodulation is fixed, (19) in the RLS method can be calculated offline, which are not included in Table I.

2) Non-recursive methods, including the quadrature, DFT and FFT methods

If $M=N / 2$ and the frequencies are equally-spaced, the quadrature demodulation method requires the same number of multiplications and additions as the DFT method. In the quadrature demodulation method, the number of multiplications and additions can be reduced when less frequency components are required to be measured. The FFT method requires much less multiplications and additions than the DFT method. Here, a radix-2 FFT method is used. 


\section{Comparision of Computating Time}

This paper aims to implement the demodulation methods in a DSP or FPGA for online demodulation. Then the operations in Table I should be executed in time so that the demodulation results can be provided after $N$ samples obtained by the ADC. In special, the multiplication operations take the most time and hardware resources. Therefore, the computing time of the methods should be carefully considered, especially when the hardware resources are limited.

Taking the implementation in the provided DSP as an example, one multiplication or addition operation takes four clock periods. The clock frequency of the DSP is $200 \mathrm{MHz}$. The DSP used in this paper includes two multiplying units, which means that two multiplication operations can be executed synchronously within four clock periods, i.e. $20 \mathrm{~ns}$. If the sampling frequency is $1 \mathrm{MHz}$, the time interval between two adjacent samples is $1 \mu \mathrm{s}$. Assume that the number of samples in a complete signal period is 100 , i.e. $N=100$. Then the number of multiplication operations up to 10000 can be executed in $100 \mu \mathrm{s}$. When $M=3$ and $N=100$, the number of multiplication operations and the computing time of the methods are shown in Table II.

TABLE II

COMPARISON OF NUMBERS OF MULTIPLICATIONS AND COMPUTING TIME AMONG DIFFERENT METHODS WHEN $M=3$ AND $N=100$

\begin{tabular}{cccc}
\hline \hline Methods & & $\begin{array}{c}\text { Number of } \\
\text { multiplications }\end{array}$ & $\begin{array}{c}\text { Computing } \\
\text { time }(\mu \mathrm{s})\end{array}$ \\
\hline Non-recursive & QD & 600 & 6 \\
demodulation & DFT & 10000 & 100 \\
& FFT & 448 & 4.48 \\
\hline Recursive & RLS & 9100 & 91 \\
demodulation & IF & 600 & 6 \\
\hline \hline
\end{tabular}

It can be seen that the IF method requires the same computing time as the quadrature demodulation method. When the sampling frequency is fixed, the numbers of multiplications and additions required by the RLS and IF methods can be reduced with less samples in partial period. However, the numbers of multiplications and additions required by the quadrature demodulation method, the DFT method or the FFT method cannot be reduced. In other words, the recursive demodulation methods are more flexible.

\section{EXPERIMENT AND RESULTS}

\section{A. Experiment}

The proposed recursive demodulation methods were implemented in a test circuit for multi-frequency signals to evaluate their performance in real applications. The schematic diagram and photo of the circuit are shown in Fig. 2 (a) and (b). An excitation signal is generated using a digitalto-analog converter (DAC) based on the direct digital synthesis technique, which contains three sinusoidal components, i.e. 10,20 and $30 \mathrm{kHz}$. The generated voltage is sampled by an ADC (Analog Device). The samples are transmitted to the DSP and FPGA, and demodulated by the aforementioned demodulators. The demodulated data are transmitted to the PC via an USB device. The ADC operates at a sampling rate of $1 \mathrm{MHz}$, meaning that there are 100, 50 and 33 sample points per period for the 10,20 and $30 \mathrm{kHz}$ sinusoidal components, respectively. The sampled data were demodulated by the abovementioned demodulators. For each demodulation process, the first 6 up to 300 samples are used to output the amplitudes and phases of the sinusoidal components. The demodulation process is repeated for 100 times to obtain 100 amplitudes and 100 phases for each update and each component.

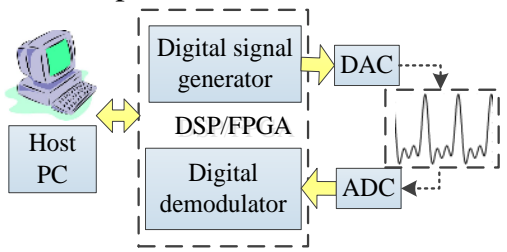

(a) schematic diagram

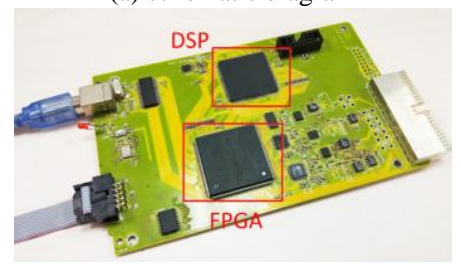

(b) photo

Fig.2 Test circuit for experiment

\section{B. Results}

As analyzed above, regardless of the hardware resource consumption, the quadrature demodulation method can be used to represent the non-recursive methods and compare with the recursive demodulation method. The multifrequency signal to be demodulated is shown in Fig. 3 (a). The variation of the averaged values of the demodulated amplitudes and phases with the number of samples is shown in Fig. 3 (b) and (c), respectively. The proposed demodulator can start to output the amplitude and phase values of the three sinusoidal components using the first six consecutive samples although the accuracy is very low. The demodulated amplitude and phase values can quickly approach their steady values, i.e. $0.43,0.35$ and $0.26 \mathrm{~V}$, and $32.2,49.3$ and 66.4 degrees, after about 25 updates. The variation of the relative error and standard deviation of the amplitudes with the number of samples is depicted in Fig. 4(a) and (b), respectively. The relative error can be calculated by

$$
e=\frac{v-v_{r}}{v_{r}} \text {. }
$$

where $v$ is the demodulated value and $v_{r}$ is the reference value.

From Fig. 4, it can be seen that when the number of samples becomes larger, the relative error and the standard deviation becomes lower. The relative error and standard deviation of the demodulated amplitudes of the three components when using different numbers of samples and different demodulators are shown in Table III. Let's define the multiples of signal period as

$$
P_{m}=\frac{N f_{m}}{f_{s}}, m=1,2,3 .
$$


TABLE III

RELATIVE ERROR AND STANDARD DEVIATION OF DEMODULATED AMPLITUDES USING DiFFERENT METHODS AND DIFFERENT NUMBERS OF SAMPLES

\begin{tabular}{|c|c|c|c|c|c|c|c|c|c|c|}
\hline & \multirow[b]{2}{*}{$\begin{array}{c}\text { Number of } \\
\text { samples }(N)\end{array}$} & \multicolumn{3}{|c|}{$10 \mathrm{kHz}$ component } & \multicolumn{3}{|c|}{$20 \mathrm{kHz}$ component } & \multicolumn{3}{|c|}{$30 \mathrm{kHz}$ component } \\
\hline & & $P_{1}$ & $\begin{array}{c}\text { Relative } \\
\text { error }\end{array}$ & $\begin{array}{c}\text { std } \\
(\mathrm{mV})\end{array}$ & $P_{2}$ & $\begin{array}{c}\text { Relative } \\
\text { error }\end{array}$ & $\begin{array}{c}\text { std } \\
(\mathrm{mV})\end{array}$ & $P_{3}$ & $\begin{array}{c}\text { Relative } \\
\text { error }\end{array}$ & $\begin{array}{c}\text { std } \\
(\mathrm{mV})\end{array}$ \\
\hline Quadrature method & 100 & 1 & $0.02 \%$ & 0.27 & 2 & $0.01 \%$ & 0.17 & 3 & $0.03 \%$ & 0.19 \\
\hline Recursive & 100 & 1 & $0.02 \%$ & 0.27 & 2 & $0.01 \%$ & 0.17 & 3 & $0.03 \%$ & 0.19 \\
\hline demodulation & 50 & 0.5 & $0.15 \%$ & 0.72 & 1 & $0.20 \%$ & 1.08 & 1.5 & $-0.01 \%$ & 0.46 \\
\hline method & 33 & 0.33 & $-0.20 \%$ & 4.9 & 0.66 & $0.64 \%$ & 4.83 & 1 & $0.09 \%$ & 7.47 \\
\hline
\end{tabular}
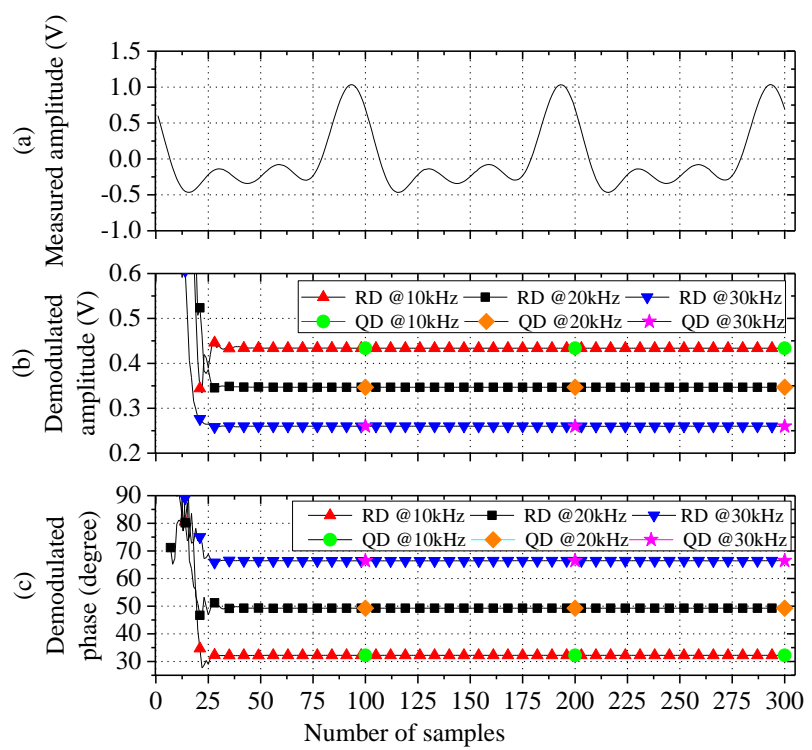

Fig. 3 (a) Multi-frequency signal to be demodulated; Averaged values of demodulated (b) amplitudes and (c) phases against number of samples $(Q D$ quadrature demodulation, $R D$--recursive demodulation)
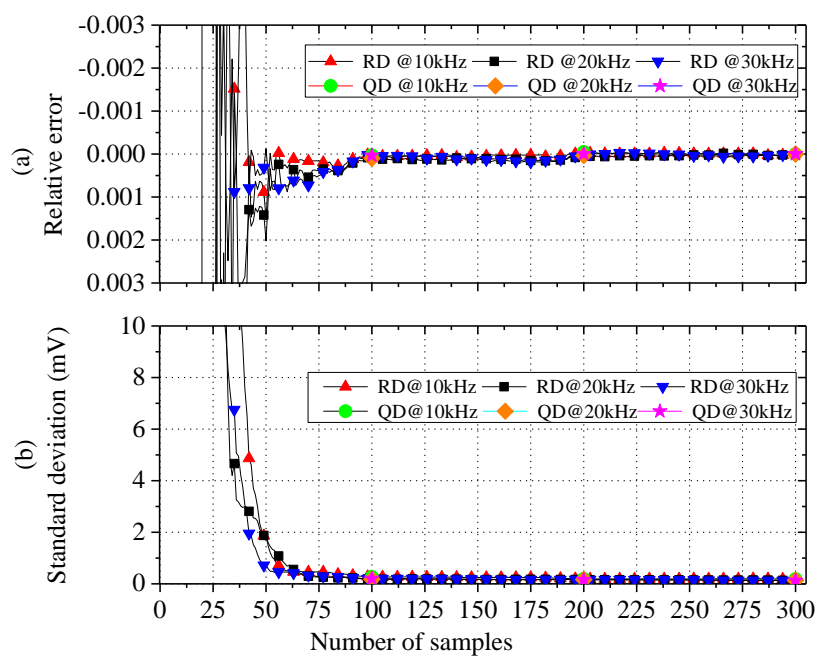

Fig. 4 Relative error (a) and standard deviation (b) of demodulated amplitudes against number of samples

According to Table III, when 100 samples are used by the recursive demodulation method, equivalent to one, two, three periods of the 10,20 and $30 \mathrm{kHz}$ components, the relative error are $0.02 \%, 0.01 \%$ and $0.03 \%$, respectively. The recursive demodulation method can generate the same results as the quadrature demodulation method when the sampled data cover exactly integer periods of the signal.
When the number of samples is fewer than 100, i.e. one complete period of the $10 \mathrm{kHz}$ sinusoid, the quadrature demodulation method cannot output any demodulation result, while the recursive demodulation method can still work well although the accuracy is a little lower. The more the samples, the lower the standard deviation. Therefore, the recursive demodulation method can not only generate continuous demodulation result for each sinusoidal component with each new sample, but also is equivalent to the quadrature demodulation method when samples of one complete or multiple periods of all frequency components are used. In addition, trade-offs between the demodulation speed and standard deviation can be obtained by the recursive demodulation method, offering greater flexibility to dynamic measurement.

\section{C.Discussions}

In this paper, the amplitudes of the frequency components are set to be constant to evaluate the demodulation accuracy of the proposed demodulator. When the demodulator is used to measure rapidly-changing signals, the demodulation process is started again after one measurement to obtain the amplitude and phase values in a new cycle. Therefore, the capability to detect the rapidly-changing signal characteristics of the proposed demodulator can be improved by acquiring less samples in one demodulation process. There are some limitations of the proposed demodulator. If the frequencies of the signal to be demodulated are very close, more samples are required to ensure the demodulation accuracy. In addition, the proposed demodulator is mainly effective to suppress the white noises. Future work will focus on the demodulation of the signal contaminated with colored noises.

\section{V.CONCLUSIONS}

This paper presents a recursive demodulator for real-time and fast demodulation of multiple sinusoids in the active measurement applications. Experimental results show that:

1) The proposed demodulator does not require the sampled data covering exactly integer periods of each constituent sinusoidal component of the signal and can continuously output demodulation results with each new sample. The demodulation speed can be greatly improved by using a shorter sample series but tradeoffs must be made between the speed and accuracy; 2) The proposed recursive demodulator is resource-saving when implemented on a DSP or FPGA, which only requires $2 M$ operations of multiplication for each update.

The proposed recursive demodulator is suitable for high- 
speed multi-frequency demodulation of sensor signal in the in-situ and on-line active measurement applications.

\section{REFERENCES:}

[1] S. Sun, Z. Cao, A. Huang, L. Xu, and W. Yang, "A High-Speed Digital Electrical Capacitance Tomography System Combining Digital Recursive Demodulation and Parallel Capacitance Measurement," IEEE Sens. J., vol. 17, no. 20, pp. 6690-6698, Oct. 2017.

[2] W. Yang, "Teaching phase-sensitive demodulation for signal conditioning to undergraduate students," Am. J. Phys., vol. 78, no. 9, pp. 909-915, Aug. 2010.

[3] L. Xu, S. Sun, Z. Cao, and W. Yang, "Performance analysis of a digital capacitance measuring circuit," Rev. Sci. Instrum., vol. 86, no. 5, p. 054703, May 2015.

[4] Y. Yang and J. Jia, "A multi-frequency electrical impedance tomography system for real-time 2D and 3D imaging," Rev. Sci. Instrum., vol. 88, no. 8, p. 085110, Aug. 2017.

[5] P. Kassanos, L. Constantinou, I. F. Triantis, and A. Demosthenous, "An Integrated Analog Readout for Multi-Frequency Bioimpedance Measurements," IEEE Sens. J., vol. 14, no. 8, pp. 2792-2800, Aug. 2014.

[6] F. Villa, A. Magnani, G. Merati, and P. Castiglioni, "Feasibility of Long-Term Monitoring of Multifrequency and Multisegment Body Impedance by Portable Devices," IEEE Trans. Biomed. Eng., vol. 61, no. 6, pp. 1877-1886, Jun. 2014.

[7] W. Yin, S. J. Dickinson, and A. J. Peyton, "A multi-frequency impedance analysing instrument for eddy current testing," Meas. Sci. Technol., vol. 17, no. 2, pp. 393-402, Feb. 2006.

[8] L. S. Rosado, P. M. Ramos, and M. Piedade, "Real-Time Processing of Multifrequency Eddy Currents Testing Signals: Design, Implementation, and Evaluation," IEEE Trans. Instrum. Meas., vol. 63, no. 5, pp. 1262 1271, May 2014.

[9] J. Qin, C. E. Stroud, and F. F. Dai, "FPGA-Based Analog Functional Measurements for Adaptive Control in Mixed-Signal Systems," IEEE Trans. Ind. Electron., vol. 54, no. 4, pp. 1885-1897, Aug. 2007.

[10]D. Chen, W. Yang, and M. Pan, "Design of Impedance Measuring Circuits Based on Phase-Sensitive Demodulation Technique," IEEE Trans. Instrum. Meas., vol. 60, no. 4, pp. 1276-1282, Apr. 2011.

[11]M. Min, T. Parve, A. Ronk, P. Annus, and T. Paavle, "Synchronous Sampling and Demodulation in an Instrument for Multifrequency Bioimpedance Measurement," IEEE Trans. Instrum. Meas., vol. 56, no. 4, pp. 1365-1372, Aug. 2007.

[12] A. J. Wilson, P. Milnes, A. R. Waterworth, R. H. Smallwood, and B. H. Brown, "Mk3.5: a modular, multi-frequency successor to the Mk3a EIS/EIT system," Physiol. Meas., vol. 22, no. 1, p. 49, 2001.

[13]L. Sujbert and G. Orosz, "FFT-Based Spectrum Analysis in the Case of Data Loss," IEEE Trans. Instrum. Meas., vol. 65, no. 5, pp. 968-976, May 2016.

[14] W. Wang, D. Ding, N. Chen, F. Pang, and T. Wang, "Quasi-Distributed IFPI Sensing System Demultiplexed With FFT-Based Wavelength Tracking Method," IEEE Sens. J., vol. 12, no. 9, pp. 2875-2880, Sep. 2012.

[15]D. Zheng, S. Zhang, S. Wang, C. Hu, and X. Zhao, "A Capacitive Rotary Encoder Based on Quadrature Modulation and Demodulation," IEEE Trans. Instrum. Meas., vol. 64, no. 1, pp. 143-153, Jan. 2015.

[16]Z. Cui, H. Wang, Z. Chen, Y. Xu, and W. Yang, "A high-performance digital system for electrical capacitance tomography," Meas. Sci. Technol., vol. 22, no. 5, p. 055503, May 2011.

[17] A. Norouzpour-Shirazi, M. F. Zaman, and F. Ayazi, "A Digital Phase Demodulation Technique for Resonant MEMS Gyroscopes," IEEE Sens. J., vol. 14, no. 9, pp. 3260-3266, Sep. 2014.

[18]P. Kassanos, I. F. Triantis, and A. Demosthenous, "A CMOS Magnitude/Phase Measurement Chip for Impedance Spectroscopy," IEEE Sens. J., vol. 13, no. 6, pp. 2229-2236, Jun. 2013.

[19]L. Xu, H. Zhou, and Z. Cao, "A recursive least squares-based demodulator for electrical tomography," Rev. Sci. Instrum., vol. 84, no. 4, p. 044704, Apr. 2013.

[20] S. Sun, L. Xu, Z. Cao, H. Zhou, and W. Yang, "A high-speed electrical impedance measurement circuit based on information-filtering demodulation,” Meas. Sci. Technol., vol. 25, no. 7, p. 075010, Jul. 2014.
[21]S. Haykin, Adaptive filter theory. Publishing House of Electronics Industry ;Pearson, 2002.

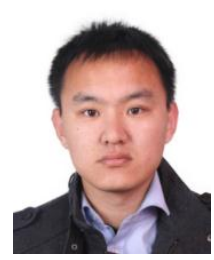

Shijie Sun (M'17) received the B.Sc. degree in automation from Tianjin University, Tianjin, China, in 2011 and received the Ph.D. degree in measurement technology and instruments from Beihang University, Beijing, China, in 2017. He is currently a post-doctor in Beihang University, Beijing, China. His current research interests include electrical tomography and digital signal processing.

Lijun Xu (M'04-SM'04) received the B.Sc., M.Eng., and $\mathrm{Ph} . \mathrm{D}$. degrees in electrical engineering and instrumentation from Tianjin University, Tianjin, China, in 1990, 1993, and 1996, respectively. From 1997 to 2001, he was an Associate Professor with the School of Electrical Engineering and Automation, Tianjin University.

From January 2002 to December 2004, he was a Research Fellow with the University of Greenwich at Medway, Chatham, U.K., and the University of Kent, Canterbury, U.K. From December 2004 to April 2006, he was a Higher Scientific Officer with the Department of Physics, Institute of Cancer Research, University of London, London, U.K. He is currently a Professor with the School of Instrument Science and Opto-Electronic Engineering, Beihang University, Beijing, China. He has authored or coauthored more than 260 publications. His current research interests include tomographic imaging, scanning imaging and dynamic process monitoring. He was elected as a Chang-Jiang Scholars Program Professor by the Ministry of Education, Ministry of Organisation, China, in 2014.

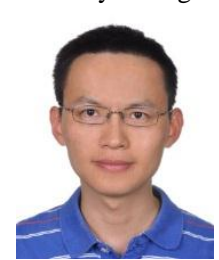

Zhang Cao (M'10) received the B.Sc. degree (with distinction) in automation and the M.Eng. and Ph.D. degrees (with distinctions) in measurement technology and automatic devices from Tianjin University, Tianjin, China, in 2003, 2005, and 2008. He is currently a professor with the School of Instrumentation Science and Opto-Electronic Engineering, Beihang University, Beijing, China. His interests include process tomography, multiphase flow measurement, and inverse problems.

Jiangtao Sun (M'12) received the B.Eng. and M.Eng

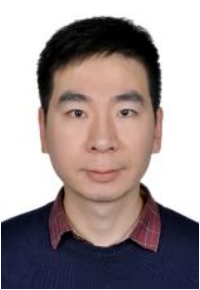
degree in measurement and control technology and instrument from Xidian University, Shaanxi, China, in 2008 and 2010, respectively, and the Ph.D. degree in electrical and electronic engineering from The University of Manchester, Manchester, U.K., in 2014. He is currently an associate professor in Instrumentation Science with Beihang University, Beijing, China. His research interests include electrical capacitance tomography and electrical impedance tomography for industrial and biomedical applications.

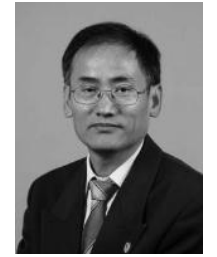

Wuqiang Yang (SM'05-F'12) received the B.Eng. (Hons.), M.Sc., and Ph.D. (Hons.) degrees from Tsinghua University, Beijing, China, in 1982, 1985, and 1988, respectively. After three years as a Lecturer with Tsinghua University, he joined The University of Manchester, Manchester, U.K., in 1991, where he has been a Professor since 2005

He has been invited by many universities, research institutions, and international conferences worldwide to give lectures, seminars, and keynotes. He has authored 300 papers and holds 10 patents. His current research interests include electrical capacitance tomography and multiphase flow measurement. He is an Associate Editor of the IEEE Tran. on Instru. and Meas., an Editorial Board Member of six other journals, and a Guest Editor of several journal special issues, and reviews papers for 40 professional journals, including six IEEE Transactions. Since 2002, he has been in Who's Who in the World. He is currently a Distinguished Lecturer with the IEEE Instru. and Meas. Soc.. 